\title{
- Effect of Ion-Molecule Collisions in the Vacuum Chamber of an Electrospray Time-of- Flight Mass Spectrometer on Mass Spectra of Proteins
}

\author{
I. V. Chernushevich, ${ }^{*}$ A. N. Verentchikov, ${ }^{\dagger}$ W. Ens, and K. G. Standing \\ Physics Department, University of Manitoba, Winnipeg, Canada
}

Electrospray ionization spectra of positive multiply charged ions of several proteins with molecular weight from 8565 to 339,100 u were recorded at different pressures of residual gas in the vacuum chamber of an electrospray time-of-flight mass spectrometer. The pressure was varied in the range $(0.2$ to 5$) \times 10^{-6}$ torr. The effect of pressure was found to be significant even for the lightest protein investigated (ubiquitin), which resulted in a decrease of both sensitivity and resolution. Investigations of the arrival-time distributions and the energy distributions of ions showed that collision-induced dissociation (CID) of the protein ions in the drift region is the main process responsible for the effect. Several CID cross sections of proteins were estimated from a series of mass spectra recorded at different pressures in the reflecting mode: $1150 \AA^{2}$ for cytochrome $c$ (averaged over charge states $z=14-18), 800 \AA^{2}$ for lysozyme $(z=8-10), 1840 \AA^{2}$ for apomyoglobin $(z=12-25), 800 \AA^{2}$ for holomyoglobin $(z=8)$, and $2500 \AA^{2}$ for carbonic anhydrase II $(z=22-35)$. Several experiments with large proteins in their native conformations and low charge states $(\mathrm{m} / \mathrm{z} \leq$ $10,000)$ demonstrate that these ions are less sensitive to high residual gas pressure. (J Am Soc Mass Spectrom 1996, 7, 342-349)

$\mathrm{T}$ The advent of electrospray ionization (ESI) and matrix-assisted laser desorption (MALDI) in the 1980 s provided a significant increase in the maximum mass detectable by mass spectrometry. This limit has been changing by an order of magnitude every 10 years or so [1], but the megau range predicted for the year 2000 has already been reached [2, 3].

Molecules of very high mass may have large collision cross sections, as indicated by recent measurements for proteins at low electronvolt energies, which yielded values of 800 (for motilin, $2699 u$ ) to $14,000 \AA^{2}$ (for albumin, 66,431 u) [4]. Earlier measurements of cross sections for collision-induced dissociation (CID) of $20-\mathrm{keV}$ ions produced by MALDI also gave values of $\sigma_{\mathrm{CID}}$ up to $1410 \AA^{2}$ for collisions of singly charged cytochrome $c$ ions with nitrogen molecules [5]. Collisions with residual gas molecules may therefore be expected to give significant effects at pressures commonly used in mass spectrometers. This factor is well

\footnotetext{
Address reprint requests to Dr. Kenneth G. Standing, Physics Department, University of Manitoba, Winnipeg, Manitoba R3T 2N2, Canada. - On leave from the Institute of Energy Problems of Chemical Physics, Russian Academy of Sciences, Chemogolovka, Moscow region, 142432, Russia.

${ }^{\dagger}$ Present address: PerSeptive Biosystems, Framingham, MA 01701.
}

known but the consequences of a high background pressure often are underestimated. A strong pressure dependence was demonstrated recently for MALDI [6], where ions are ejected directly into vacuum. The influence of pressure can be even more relevant in ESI: ions enter the vacuum chamber from atmosphere through a series of orifices, so there is always a compromise between better vacuum and better sensitivity. The goal of the present study was to investigate the effects of the residual pressure, to find out what processes are responsible for the main changes in spectra and, if possible, to find some ways to improve the situation. Some of the measurements described here were presented at the 42nd ASMS Conference [7].

\section{Experimental}

All the results presented herein were obtained with an electrospray ionization time-of-flight (ESI-TOF) mass spectrometer described previously [8]. In brief, the instrument (Figure 1) uses the method of orthogonal ion injection into a TOF mass spectrometer that was introduced by Dodonov et al. $[9,10]$ for electrosprayed ions. Ions produced at atmospheric pressure are transported through three stages of differential pumping to storage region 6 , where they continue their motion 


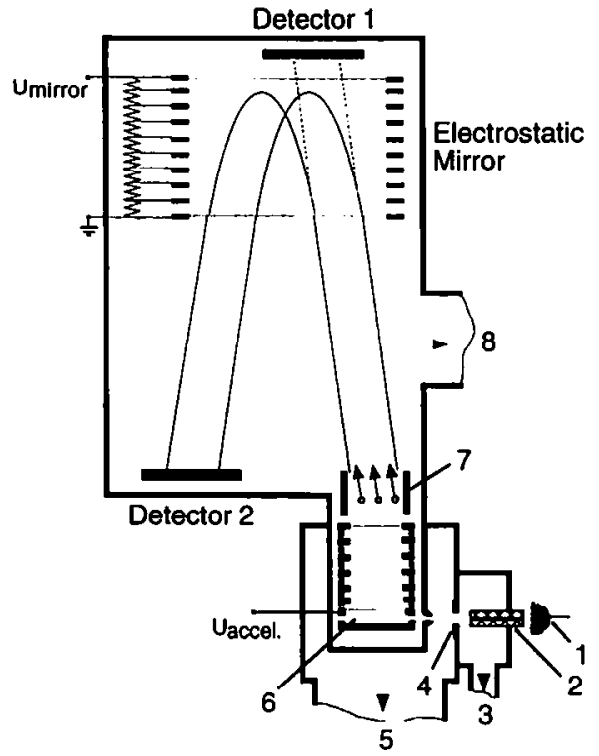

Figure 1. Schematic diagram of the reflecting TOF mass spectrometer with orthogonally injected electrospray ions. 1, electrospray capillary; 2 , heated metal capillary; $3,12-\mathrm{L} / \mathrm{s}$ rotary pump; 4, skimmer; 5, 450-L/s turbopump; 6, ion storage region; 7 , deflection plates; $8,1000-\mathrm{L} / \mathrm{s}$ cryopump.

with an energy of $\sim 10 \mathrm{eV}$. Extraction pulses are applied to the storage region gap at a repetition rate of several kilohertz, and ions are accelerated to $\sim 4000-\mathrm{eV}$ energy per charge in the direction of the ion mirror. The spectrometer may be run in the linear mode, with the mirror voltage off and ions observed in detector 1 . However, the instrument is normally operated in the reflecting mode, which gives better compensation for the initial spatial and energy spreads of the ions. In the latter mode, ions reflected by the mirror traverse the second leg of the drift region and are observed in detector 2. Each detector consists of two $40-\mathrm{mm}$ diameter microchannel plates in a chevron configuration. The detector signal passes to a preamplifier and a constant fraction discriminator and is then registered by an Orsay time-to-digital converter (Model CTN-M2, Institute de Physique Nucléar, B.P. No. 1, Orsay Cedex F-91406, France).

The pressure in the vacuum chamber was varied in the range $(0.2-5) \times 10^{-6}$ torr by partial closure of the valve that separates the main chamber from the cryopump. The residual gas is assumed to be mostly nitrogen, which is used for the gas curtain in the ion source. The partial pressure of water vapor is probably smaller, because the cryopump has high efficiency to pump $\mathrm{H}_{2} \mathrm{O}$, and the whole vacuum chamber is baked constantly to $\sim 50^{\circ} \mathrm{C}$. Mass spectra were recorded in both linear and reflecting modes in the present study. Switching from one mode to another was done by changes in two potentials only: the potential of the ion mirror and the voltage of the extraction pulse responsible for the time focusing of ions at the plane of the corresponding detector. The effective path length is $150 \mathrm{~cm}$ in the linear mode and $284 \mathrm{~cm}$ in the reflecting mode.

\section{Chemicals}

Solutions of proteins with concentrations $\sim 10^{-5} \mathrm{M}$ were prepared with deionized water $(49 \%)$, reagent grade methanol (49\%), and glacial acetic acid (2\%), unless indicated otherwise. Ubiquitin (from bovine red blood cells), cytochrome (from horse heart), lysozyme (from chicken egg white), myoglobin (from horse skeletal muscle), carbonic anhydrase II (from human erythrocytes), and albumin (from bovine serum) were obtained from Sigma Chemical Co. (St. Louis, MO) and were used without further purification. Transferrin (apo-, from human plasma) was obtained from Calbiochem (San Diego, CA). Spectra recorded from an unpurified solution of the last compound showed a series of adducts of the form $(M+98 n, n=1-10)$, consistent with the presence of sulfate or phosphate impurities. To remove the impurities, barium acetate solution was added [11] to a $5 \times 10^{-6}-\mathrm{M}$ transferrin solution in water-methanol to give a final salt concentration of $3 \times 10^{-4} \mathrm{M}$. Catalase HP II from E. coli was obtained from P. C. Loewen (Microbiology Department, University of Manitoba) and was purified by centrifugal filtration of the aqueous solution by using ultrafree-MC filters with a 30,000-u molecular weight cutoff (Millipore Co., Bedford, MA). High purity ammonium acetate (99.999\%; Aldrich Chemical Co., Milwaukee, WI) was used for buffers.

\section{Results and Discussion}

Electrospray ionization spectra of positive multiply charged ions of several proteins ( 8565 to $79,550 \mathrm{u}$ ) were recorded at different residual gas pressures in the vacuum chamber of the ESI-TOF mass spectrometer. The effects on the ESI-TOF spectrum of cytochrome $c$ (shown in Figures 2 and 3) are representative. In both cases the peak heights are considerably reduced - by a factor $\sim 2.5$ in Figure 2 and by a factor $\sim 10$ in Figure 3 . In the linear mode (Figure 2) the peaks become much wider while the peak area remains nearly the same; in the reflecting mode (Figure 3) the peaks become smaller but remain sharp and the background ion current increases between the peaks. Similar effects were observed for other proteins. In general, the effect increases with protein molecular weight, but was observed clearly even for ubiquitin (8565 u), the lightest protein investigated.

Several types of ion-molecule processes could be responsible for the observed changes in the spectra: (1) elastic collisions; although the collision is elastic the incident ion loses the recoil energy given to the target molecule; (2) inelastic collisions with the loss of a small neutral or charged particle (e.g., a proton); (3) inelastic collisions that result in dissociation of the protein into two fragments of comparable mass and charge. The last process would seem to be the most probable due to the large energy of the ions in the drift tube $(\sim 4$ $\mathrm{keV}$ per charge) and for the same reason the cleav- 


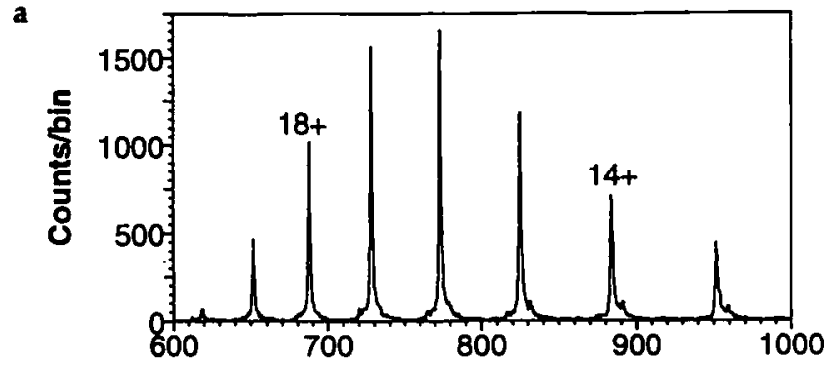

b

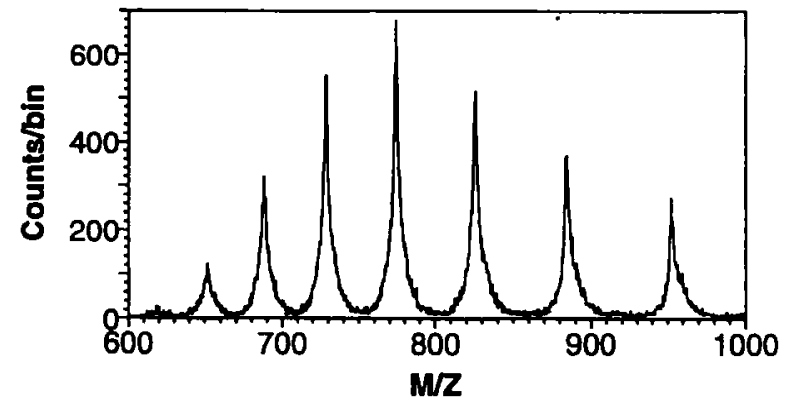

Figure 2. ESI mass spectra of horse heart cytochrome $c(12,360$ u) recorded at two different pressures $p$ in the vacuum chamber in the linear mode: (a) $p=0.75 \times 10^{-6}$ torr; (b) $p=4.9 \times 10^{-6}$ torr. Each spectrum was recorded in $1 \mathrm{~min}$.

age is probably random. To determine experimentally which of these processes causes changes in the spectra, detailed arrival-time distributions and energy distributions of the ions (measured by using the ion mirror as an energy filter) were recorded. All proteins were electrosprayed from $2 \%$ acetic acid-water-methanol (1:1) solutions, and their charge states corresponded to denatured forms of the proteins.

Arrival-time distributions of cytochrome $c$ in the linear mode (in the form of deconvoluted spectra) for

a

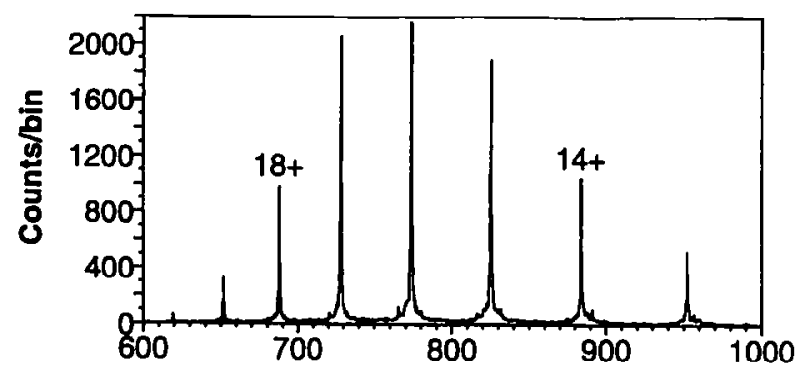

b

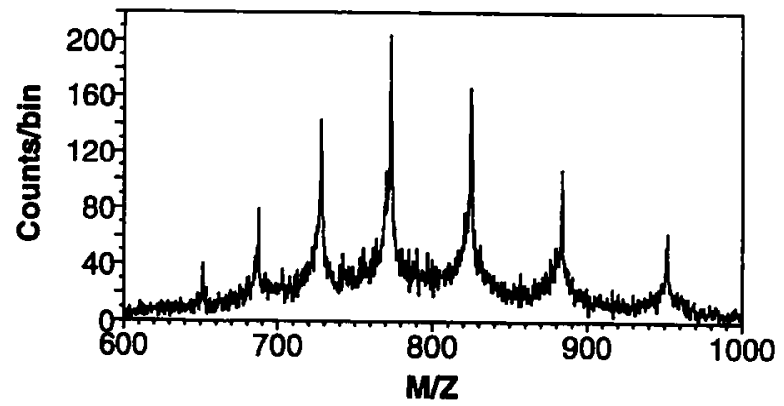

Figure 3. ESI mass spectra of horse heart cytochrome $c(12,360$ u) recorded at two different pressures in the vacuum chamber in the reflecting mode: (a) $p=0.8 \times 10^{-6}$ torr; (b) $p=4.1 \times 10^{-6}$ torr. Each spectrum was recorded in $1 \mathrm{~min}$.

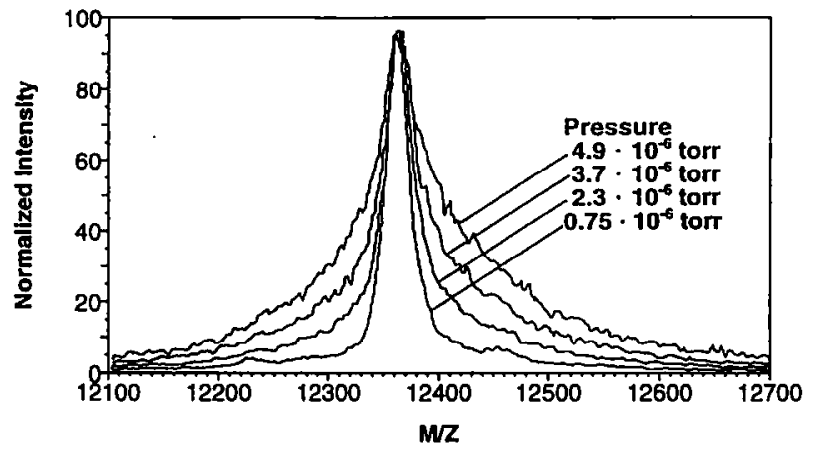

Figure 4. Deconvoluted ESI mass spectra of cytochrome $c$ recorded in the linear mode at different pressures in the vacuum chamber. Charge states $z=14-18$ were used in the deconvolution.

four different pressures are shown in Figure 4. It is easy to see that the peak broadens substantially in both directions as the pressure increases. The dependence of peak width on pressure in the linear mode is shown in Figure 5 for five proteins: ubiquitin, cytochrome $c$, apo-myoglobin, carbonic anhydrase II, and albumin. In contrast to the considerable change in peak width with pressure, the effect on peak position is rather small (Figure 6).

Energy distributions of cytochrome $c$ ions were measured in the reflecting mode by changing the ion mirror potential $\left(U_{\text {mirror }}\right)$ for values in the vicinity of the accelerating potential $\left(U_{\text {accel. }}\right.$.). The number of ions $N$ that arrive at the detector was measured within a wide time window that corresponds to the parent and fragment ions of interest. Thus the integral curves of energy per charge are obtained for different vacuum chamber pressures (Figure 7a), and the first derivative $d N / d U_{\text {mirror }}$ gives us the energy per charge distributions (Figure $7 \mathrm{~b}$ ). Without collisions the ion energy is determined by the accelerating potential of $4000 \mathrm{~V}$ plus the value of the extraction pulse $200 \mathrm{~V}$ applied to the

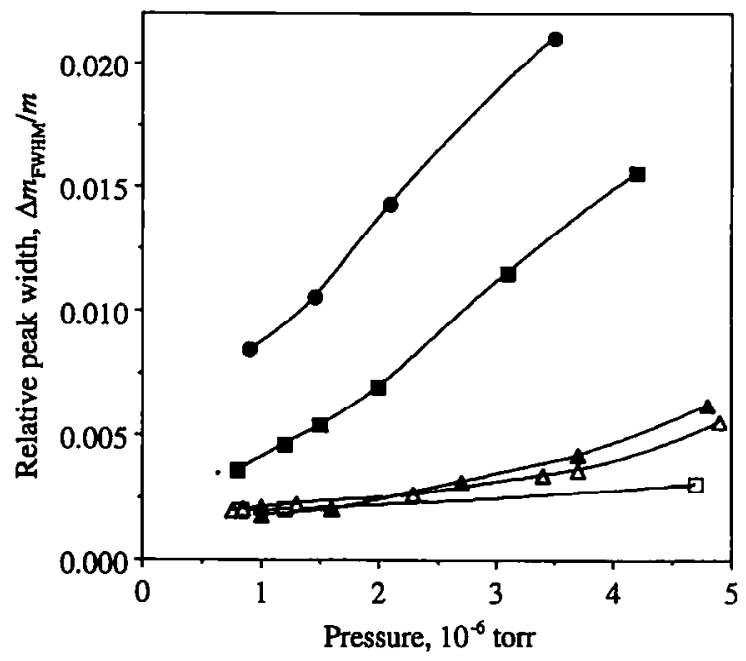

Figure 5. Relative peak width measured at half-height (reciprocal resolution) of five proteins as a function of pressure in the vacuum chamber (linear mode): $\square$, ubiquitin; $\Delta$, cytochrome; $\Delta$, myoglobin; $\boldsymbol{\Pi}$, carbonic anhydrase; $\boldsymbol{O}$, albumin. 


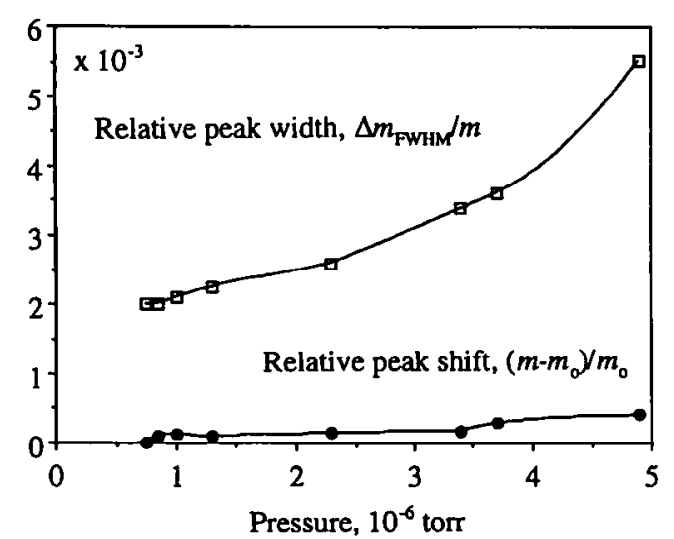

Figure 6. Relative peak width (full width at half-maximum) and shift (measured above half-height) of cytochrome $c$ determined from Figure 4 as a function of the pressure.

ion storage region in Figure 1. Ions may therefore have an energy spread of up to $200 \mathrm{eV}$ per unit charge due to their spatial spread in the ion storage region. The curves for the lowest pressure in Figure 7 verify this. For higher pressures the number of counts measured for $U_{\text {mirror }}>4200 \mathrm{~V}$ increases, and both high and low energy tails appear in the energy distributions.

The results shown in Figures 2-7 can be explained easily if it is assumed that "random" collision-induced dissociation of multiply charged ions into multiply charged fragments is the main process that takes place in the vacuum chamber. Due to Coulomb repulsion, fragments acquire an additional energy up to $10 \mathrm{eV}$
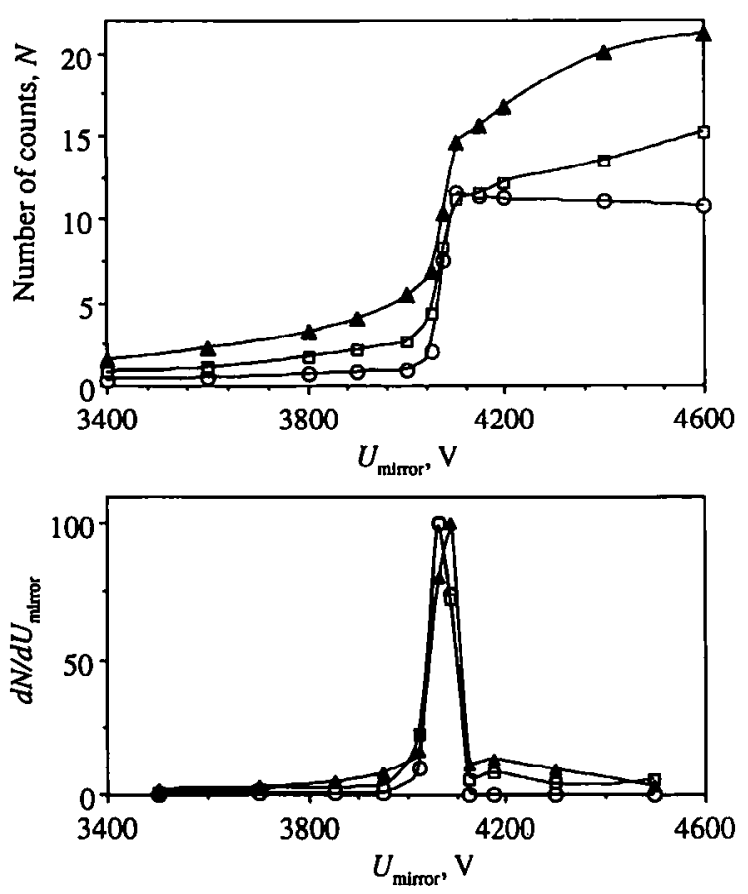

Figure 7. Energy per charge filtering by ion mirror. (a) Total number of counts $N$ (thousands) of cytochrome $c$ versus ion mirror potential at different pressures $p: 0, p=0.41 \times 10^{-6}$ torr; $\square, p=1.5 \times 10^{-6}$ torr; $\triangle, p=2.6 \times 10^{-6}$ torr. (b) Normalized first derivative of $N$.
[12] in the center of mass system with an isotropic distribution of the corresponding velocities. This would cause the tailing of peaks in both directions in the linear mode. On the other hand, elastic collisions would cause a decrease in energy that results in the broadening on the high time side only. In the reflecting mode, the ion mirror produces better separation of fragment ions from their parents, and fragment ions are scattered into a wider time window. The large number of possible fragments and their charge states, together with the poor resolution for fragments formed in the drift region, leads to the formation of a slowly changing background (Figure 3). Therefore only those parent ions that do not collide with residual gas molecules before they leave the ion mirror contribute to the peaks, which remain sharp.

The distributions measured in the reflecting mode (Figure 7) also indicate dissociation into multiply charged ions. The ion mirror filters ions according to their energy per unit charge. If the parent and fragment ions have masses $m_{\mathrm{p}^{\prime}} m_{\mathrm{f}}$ and charges $z_{\mathrm{p}}, z_{\mathrm{f}}$ correspondingly, the value of energy per unit charge of the fragment ion is equal to $z_{\mathrm{p}} m_{\mathrm{f}} U_{\text {accel. }} / z_{\mathrm{f}} m_{\mathrm{p}}$. This value may be either smaller or larger than that for the parent ion, dependent on how the mass and charge of the parent ion are divided between the fragments. Elastic collisions or small neutral losses would result only in low energy tails.

The measured distributions show that ion selection in the mirror is not likely to counter the adverse effect of collision-induced dissociation of ions on spectra because fragment ions may have same mass-to-charge ratio and energy per charge as parent ions. Nevertheless, some improvement by filtration is possible if the single ion counting technique is used to record the spectra. It is known that the secondary electron yield of ions that hit a detector increases with mass for a given velocity [13]. Thus, ions with different masses but the same mass-to-charge ratio (i.e., the same velocity) can be resolved by changing the threshold of the discriminator. This method already has been used in Uppsala and is called secondary electron resolved mass spectrometry [14]; it has been reinvented independently by several other groups [15-17]. We have found that the method is useful both to reduce low mass chemical noise in the spectra of proteins [16] and to reduce the "fragmentation" noise in the case of collision-induced dissociation of proteins. Two spectra of bovine albumin shown in Figure 8a and $b$ were recorded at low $\left(4 \times 10^{-7}\right.$ torr $)$ and high $\left(2.6 \times 10^{-6}\right.$ torr) pressures with the same threshold $(10 \mathrm{mV})$ of the constant fraction discriminator. Nearly all ions that hit the detector are recorded with this value of the threshold. An increase of the threshold to $200 \mathrm{mV}$ leads to a tremendous loss of counts (Figure 8c), but improves the quality of the mass spectrum. Note that a narrow peak at $m / z 1111$ present in Figure 8a and $b$ was not recorded with the 200-mV threshold (Figure 8c). This peak belongs to a dimer of leucine enkephalin, which 

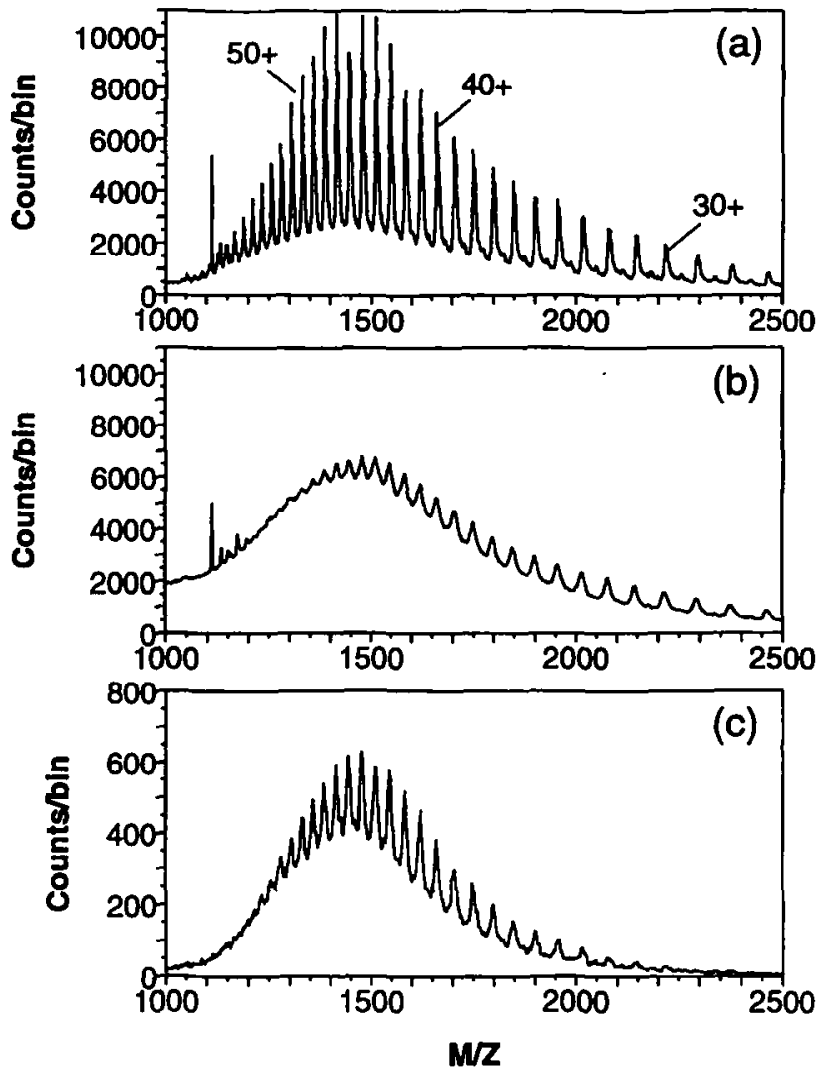

Figure 8. Mass spectra of bovine albumin: (a) $p=4 \times 10^{-7}$ torr; $(b, c) p=2.6 \times 10^{-6}$ torr. In (c) the threshold of the constant fraction discriminator was increased from 10 to $200 \mathrm{mV}$.

was used as an internal calibrant in these experiments. The spectra in Figure $8 b$ and $c$ were recorded simultaneously by using two discriminators.

It is clear from the previous discussion that for large proteins, the vacuum chamber of the mass spectrometer serves as a collision cell. This analogy can be extended: cross sections $\sigma$ for collision-induced dissociation can be estimated from a series of mass spectra recorded at different pressures in the reflecting mode. Only those ions that do not fragment before they leave the mirror contribute to the sharp peaks. The total length of this collision cell is $L=157 \mathrm{~cm}$ in our case $(98 \mathrm{~cm}$ for the first leg of the field-free region plus 59 $\mathrm{cm}$ for two times the path of ions in the mirror). The

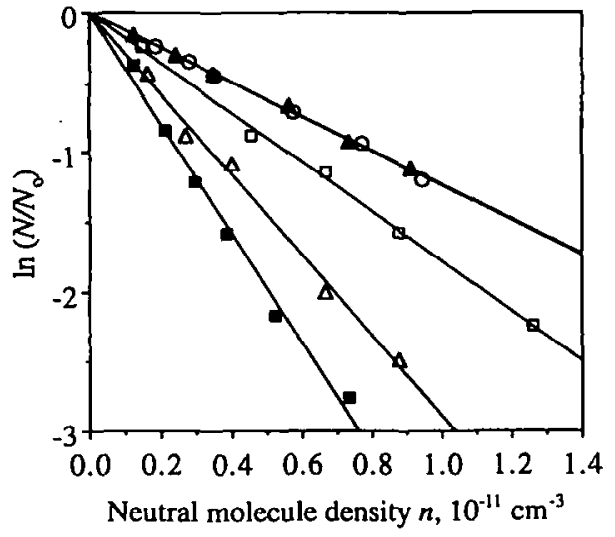

Figure 9. Logarithmic plot of relative decrease of deconvoluted peak area (measured above half-maximum) $N / N_{0}$ versus neutral molecule density $n$ in the vacuum chamber: $\square$, cytochrome; $O$, lysozyme; $\Delta$, apomyoglobin; $\Delta$, holomyoglobin; $\boldsymbol{\square}$, carbonic anhydrase.

area of the deconvoluted peak $N$ is then proportional to $\exp (-\sigma n L)$, where $n$ is the neutral molecule density. Thus, measurement of the area $N$ at different pressures enables $\sigma$ to be estimated from the slope of $\ln N$ versus $n$, as shown in Figure 9. The peak area $N$ was calculated above the half-height of the peak to minimize the contribution of fragment ions. In contrast to the MALDI experiments [5], where the relative reduction of the ion signal was measured for each value of pressure, only absolute intensities were measured in the present case. Therefore, a good long-term stability of the electrospray current (within a few percent for a single curve in Figure 9) was necessary for measurements of $\sigma$. On the other hand, the reduced ion intensities at high pressures required accumulation of each spectrum for at least $2 \mathrm{~min}$ to get appropriate statistics, and each experiment summed to about 20 $\mathrm{min}$. Thus, the time during which spectra were recorded was always a compromise between the stability of the electrospray current and good statistics. For this reason only the values of $\sigma$ averaged over several charge states are given in Table 1, which summarizes the data obtained for four proteins. Note that holomyoglobin (myoglobin with heme attached) was recorded from a 5-mM ammonium acetate aqueous solution; a typical spectrum is shown in Figure 10.

Table 1. Estimated cross sections of collision-induced dissociation $\sigma$ of protein ions at $\sim 4-\mathrm{keV}^{\mathrm{a}}$ energy per charge

\begin{tabular}{lllr}
\hline & $\begin{array}{c}\text { Molecular } \\
\text { weight (u) }\end{array}$ & \multicolumn{1}{c}{$z^{\mathrm{b}}$} & $\sigma\left(\AA^{2}\right)$ \\
\hline \hline Cytochrome $c$ (horse heart) & 12,360 & $14-18$ & 1150 \\
Lysozyme (chicken egg white) & 14,306 & $8-10$ & 800 \\
Myoglobin, apo- (horse) & 16,951 & $12-25$ & 1840 \\
Myoglobin, holo- (horse) & 17,567 & 8 & 800 \\
Carbonic anhydrase II, apo (human) & 29,023 & $22-35$ & 2500 \\
\hline
\end{tabular}

- $4 \mathrm{keV}$ is energy per charge of the ions in the field-free region; in the mirror it decreases gradually to zero.

${ }^{b}$ Charge states used for determination of $\sigma$ 


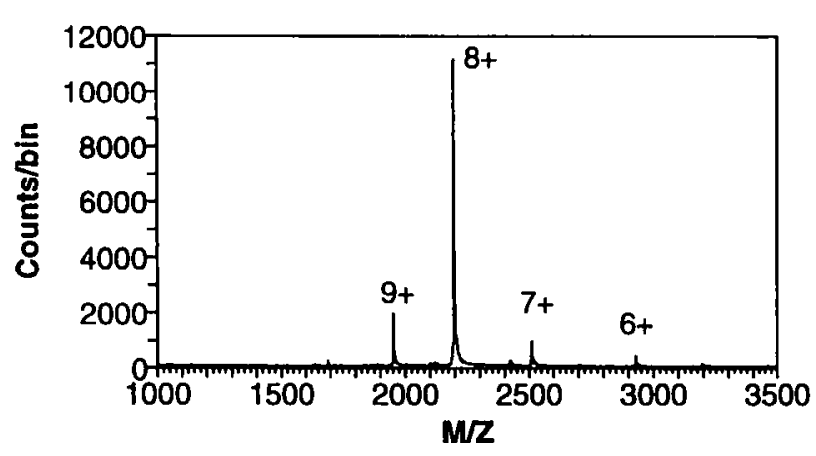

Figure 10. Mass spectrum of holomyoglobin recorded from 5-mM ammonium acetate aqueous solution.

Collision cross sections of proteins have been studied previously in $[4,5]$, as previously mentioned, but the data obtained in these studies should not be compared directly with those in Table 1, because the experimental conditions were quite different in all three cases. Cross sections for collision-induced dissociation were measured in [5] and in the present study, whereas in [4] the collisions did not lead to fragmentation. A tremendous difference in the ion energy in the quadrupole and TOF instruments (10 eV versus several kiloelectronvolts per charge) implies that the low energy experiments are described better by the Langevin model, whereas conditions in TOF mass spectrometry are probably closer to a hard sphere model. Finally, MALDI experiments and those made with ESI differ strongly in charge states and, possibly, conformations of ions. Therefore, the range of cross sections obtained for cytochrome $c$, for example (1150 $\AA^{2}$ in this study, $1410 \AA^{2}$ in [5], and from 1450 to $4310 \AA^{2}$ for different charge states in [4]), is not surprising.

To investigate the limitations the ion-molecule collisions may impose on the performance of the TOF instrument, several spectra of large proteins were recorded. The best results were achieved for human apo-transferrin, 79,550 u MW, which was sufficiently homogeneous and pure to give a resolution $m / \Delta m_{\text {FWHM }}$ close to 2000 and an experimentally determined mass within $7 \mathrm{u}$ of that measured by Feng et al. [18]. The spectrum shown in Figure 11a was recorded with the usual pressure in the vacuum chamber, $p=$ $3 \times 10^{-7}$ torr. Surprisingly, only a threefold increase in pressure (Figure 11b) is enough to deteriorate both resolution and sensitivity in the spectrum of transferrin.

Most of the studies described in the preceding text were performed with denatured proteins, but the single example of holomyoglobin demonstrates that CID cross sections may be much smaller for proteins in their native form. Similar results were obtained in [4], where cytochrome $c$ was studied from 9:1 and 1:1 water-acetonitrile solutions. At least two factors may contribute to the increased stability of native conformations: their smaller dimensions (i.e., geometrical cross sections) and a large number of intermolecular interactions, which hold the molecule together even if some bonds are broken during collisions. It is well known that more compact conformations of proteins are usually less protonated (partly due to higher Coulomb repulsion), and the data for lysozyme and myoglobin in Table 1 are in good agreement with this fact. Thus, we may say that in the first approximation the lower the charge state of a given ion (the greater the mass-to-charge ratio), the less it is affected by ion-molecule collisions. In addition to the abovementioned reasons for the increased stability of native conformations, lower charge states have smaller energy. Therefore better quality spectra of heavier proteins (biomolecules) are more likely to be recorded at higher values of mass-to-charge ratio. Several examples of studies of non-covalent associations on the ESI-TOF mass spectrometer $[3,19]$ seem to confirm this idea. The heaviest complex investigated on this instrument was catalase HP II from E. coli, a tetrameric protein with mass $339,100 \mathrm{u}$. The ions of HP II were observed at $m / z \sim 10,000$ when the complex was sprayed from 5-mM ammonium acetate solution, $\mathrm{pH}$ $\sim 6$. The peaks of the three most abundant charge states recorded at normal pressure in the vacuum chamber are shown in Figure 12a. In contrast to the previous example of transferrin, the spectrum did not change much when the pressure was increased to $1.3 \times 10^{-6}$ torr, as shown in Figure 12b. Some increase in the peak height at higher pressure is due to fluctuations of the electrospray current.

Two interesting questions were not addressed in these studies. First, it would be of interest to compare

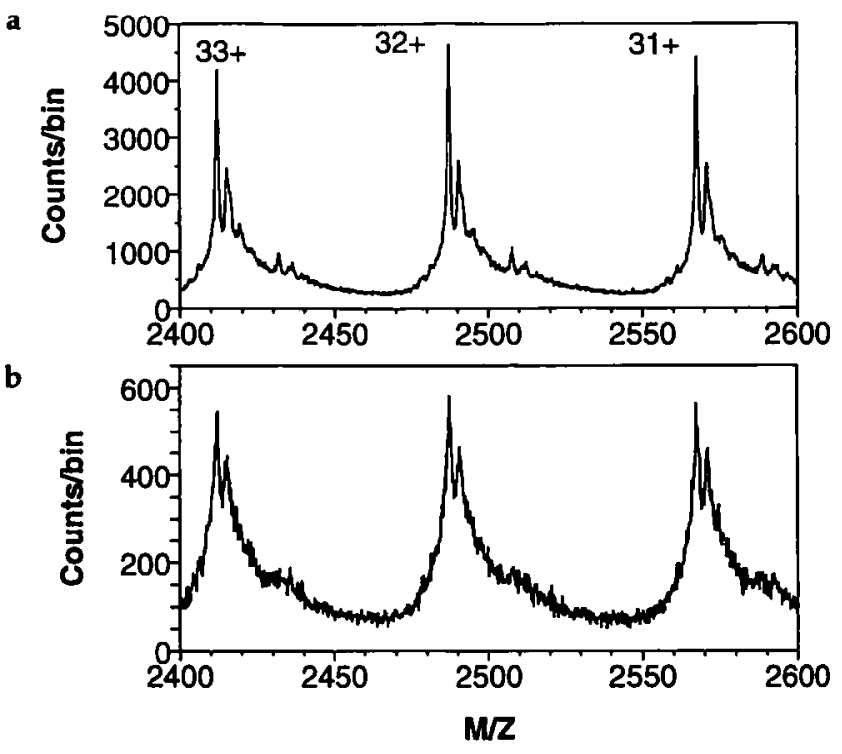

Figure 11. Segments of the mass spectra of human apo-transferrin recorded at two different pressures in the vacuum chamber for the same time interval: (a) $3 \times 10^{-7}$ torr; (b) $1.0 \times 10^{-6}$ torr. Barium acetate was added to the solution to eliminate acidic adducts $(M+98 n 1<n 10)$ present in the initial spectrum. 


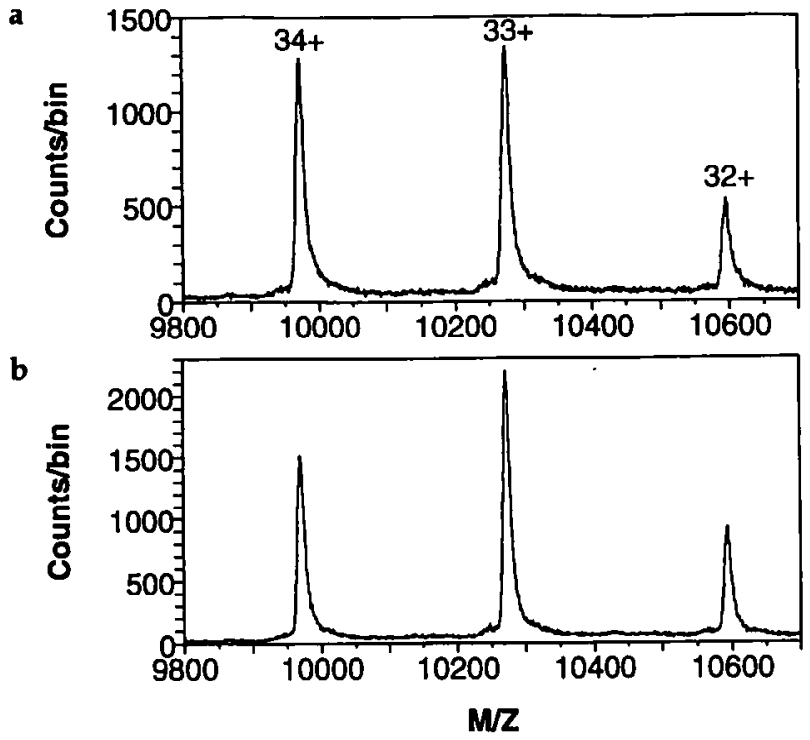

Figure 12. Segments of the ESI mass spectra of catalase HP II $(339,100 \mathrm{MW})$ recorded at two different pressures in the vacuum chamber for the same time interval: (a) $2 \times 10^{-7}$ torr; (b) $1.3 \times$ $10^{-6}$ torr.

the CID cross sections for same charge states that come from different conformations of a protein. The results of such studies could give insight into real conformations of the electrosprayed proteins in vacuum. Unfortunately, these overlapping charge states are of low abundance in the mass spectra, so it is difficult to use the data for accurate determination of cross sections, as explained previously. The second question is the influence of the initial internal excitation of ions on the CID cross sections. This influence was shown to be large both in MALDI [5], where the degree of excitation was controlled by choice of matrix, and in ESI [20], where the initial collisional excitation was produced in the atmosphere-vacuum interface. In the present study, initially "cold" ions were investigated, because the value of the declustering potential was low $(\Delta U=40 \mathrm{~V})$ in all experiments except for the spectra of catalase HP II. This value is just enough to desolvate the ions.

\section{Conclusion}

The results of these studies show that the effect of residual pressure may be significant for spectra of proteins when studied on the ESI-TOF mass spectrometer. Deterioration of spectra starts at pressure as low as $(0.5-1) \times 10^{-6}$ torr, a pressure commonly used in mass spectrometers, and affects mostly the resolution in the linear mode and the sensitivity in the reflecting mode. Investigations of the arrival-time distributions and the energy distributions of ions showed unequivocally that collision-induced dissociation of multiply charged ions into multiply charged fragments is responsible for these changes in the performance of the ESI instrument. The parent ions and the fragment ions that result from CID cannot be separated by energy filtering in the mirror, but the spectra can be partially restored by increasing the threshold of the discriminator in the ion counting mode. Several cross sections of collision-induced dissociation of protein ions at kiloelectronvolt energies were estimated from spectra recorded in the reflecting mode.

The effect of ion-molecule collisions on spectra may be extrapolated partially to other types of mass analyzers that work with high accelerating voltages, such as magnetic sector instruments. There the fragment ions that result from collisions would be rejected during analysis, so collisions would affect mostly the sensitivity.

The results of these studies demonstrate also that low charge states from native conformations of proteins or complexes are less sensitive to high residual gas pressure. The unlimited "mass-to-charge ratio" range of the ESI-TOF mass spectrometer is therefore rather useful to record the spectra of large biomolecules.

\section{Acknowledgments}

This work was supported by grants from the U.S. National Institutes of Health (GM-30605) and from NSERC, Canada.

\section{References}

1. Matsuo, T.; Sakurai, T.; Ito, H.; Wada, Y. Int. J. Mass Spectrom. Ion Processes 1992, 118/119, 635.

2. Chen, R.; Cheng, X.; Mitchel, D. W.; Hofstadler, S. A.; Wu, Q.; Rockwood, A. L.; Sherman, M. G.; Smith, R. D. Anal. Chem. 1995, 67, 1159.

3. Chernushevich, I. V.; Ens, W.; Standing, K. G.; Loewen, P. C.; Fitzgerald, M. C.; Kent, S. B. H.; Werlen, R. C.; Lankinen, M.; Tang, X.-J.; Brewer, C. F.; Saha, S. Proceedings of 43rd ASMS Conference on Mass Spectrontetry and Allied Topics, Atlanta, GA, May 21-26, 1995; p 1327.

4. Covey, T.; Douglas, D. I. Am. Soc. Mass Spectrom. 1993, 4, 616.

5. Spengler, B.; Kirsch, D.; Kaufmann, R. J. Phys. Chem. 1992, 96, 9678.

6. Schuerch, S.; Schaer, M.; Boernsen, K. O.; Schlunegger, U. P. Biol. Mass Spectrom. 1994, 23, 695.

7. Chernushevich, I. V.; Verentchikov, A. N.; Standing, K. G.; Ens, W. Proceedings of 42nd ASMS Conference on Mass Spectrometry and Allied Topics; Chicago, IL, May 29-June 3, 1994, p 989.

8. Verentchikov, A. N.; Ens, W.; Standing, K. G. Anal. Chem. 1994, 66, 126 . .

9. Dodonov, A. F.; Chernushevich, I. V.; Laiko, V. V. 12th International Mass Spectrometry Conference; Amsterdam, August 1991; Extended Abstracts, p 153.

10. Dodonov, A. F.; Chernushevich, I. V.; Laiko, V. V. In Time-ofFlight Mass Spectrometry; Cotter, R. J., Ed.; American Chemical Society: Washington, DC, 1994; Symposium Series 549, p 108.

11. Chowdhury, S. K.; Katta, V.; Beavis, R. C.; Chait, B. T. J. Am. Soc. Mass Spectrom. 1990, 1, 382. 
12. Rockwood, A. L.; Busman, M.; Smith, R. D. Int. J. Mass Spectrom. Ion Processes 1991, 111, 103.

13. Beuhler, R. J.; Friedman, L. Nucl. Instrum. Methods 1980, 170 , 309.

14. Axelsson, J.; Reimann, C. T.; Sundqvist, B. U. R. Int. J. Mass Spectrom. Ion Processes 1994, 133, 141.

15. Bondarenko, P. V.; Grant, P. G.; Macfarlane, R. D. Int. J. Mass Spectrom. Ion Processes 1994, 131, 181.

16. Standing, K. G.; Chernushevich, I. V.; Ens, W.; Verentchikov, A. N. Proceedings of $42 n d$ ASMS Conference on Mass Spectrome- try and Allied Topics; Chicago, IL, May 29-June 3, 1994; p 1148.

17. Loo, J. A.; Pesch, R. Anal. Chem. 1994, 66, 3659.

18. Feng, R.; Konishi, Y.; Bell, A. W. J. Am. Soc. Mass Spectrom. $1991,2,387$.

19. Tang, X.-J.; Brewer, C. F.; Saha, S.; Chernushevich, I. V.; Standing, K. G.; Ens, W. Rapid Commun. Mass Spectrom. 1994, $8,750$.

20. Smith, R. D.; Barinaga, C. J. Rapid Commun. Mass Spectrom. 1990, 4, 54. 